\title{
MODELING OF ANISOTROPIC GROWTH AND RESIDUAL STRESSES IN ARTERIAL WALLS
}

\author{
AnNA ZAhN ${ }^{a}$, DANIEL BALZAni ${ }^{a, b, *}$ \\ a Technische Universität Dresden, Institute of Mechanics and Shell Structures, 01062 Dresden, Germany \\ ${ }^{b}$ Dresden Center for Computational Materials Science (DCMS), 01062 Dresden, Germany \\ * corresponding author: daniel.balzani@tu-dresden.de
}

\begin{abstract}
Based on the multiplicative decomposition of the deformation gradient, a local formulation for anisotropic growth in soft biological tissues is formulated by connecting the growth tensor to the main anisotropy directions. In combination with an anisotropic driving force, the model enables an effective stress reduction due to growth-induced residual stresses. A method for the imitation of opening angle experiments in numerically simulated arterial segments, visualizing the deformations related to residual stresses, is presented and illustrated in a numerical example.
\end{abstract}

KEYWORDS: biomechanics, multiplicative anisotropic growth, residual stresses, opening angle.

\section{INTRODUCTION}

As living biological medium, arterial tissue is known to adapt to changes in its mechanobiological environment in order to improve the load-bearing capacities. Such adaptation processes originate from growth and remodeling and may be indicated by the existence of residual stresses under externally load-free conditions. Those residual stresses can be uncovered by extracting an arterial segment from the body and slicing it in radial direction. Typically, the segment contracts in axial direction and springs open to a degree which depends on various factors, for example the height of the blood pressure [1]. Being associated with a reduction of stress gradients in loaded states, residual stresses need to be included in numerical simulations to obtain a predictive stress distribution. Computational methods to reduce stress gradients by incorporating residual stresses are proposed in the literature [2 5 . These methods are however rather engineering approaches, as no biological processes are included to obtain the residual stresses.

Focusing on growth as part of the adaptation processes, the addition of material, resulting in increasing wall thicknesses and diameters, is related to a reallocation of the stresses which may therefore allow for an optimized load bearing behavior. For the purpose of modeling these effects, an anisotropic growth model based on the multiplicative decomposition of the deformation gradient into a growth tensor and a remaining elastic part is presented. In this context, different approaches for anisotropic growth in soft biological tissues have been published, see e. g. [6, 17, where the anisotropy of the growth mechanism is based on the definition of macrostructural growth directions as for instance the radial direction. Although the anisotropy of the material is taken into account, isotropic strainor stress-based quantities are used as driving forces in the evolution equations characterizing the growth tensor. Decomposing the growth tensor itself into two parts related to the main material anisotropy directions, the presented growth model establishes a relation between the growth tensor and the fibrous tissue structure, which yields a local formulation and therewith supersedes a geometry-specific definition of the growth directions. Aiming at rendering the growth and stress reduction mechanism more effective, an anisotropic stress-based driving force is formulated.

In addition, a procedure for the calculation of the deformations related to growth-induced residual stresses in numerically simulated arterial walls is developed. The entire framework is illustrated with the aid of a numerical example on an idealized arterial segment.

\section{Computational Framework}

Having been introduced in the context of biomechanics by Rodriguez et al. [8] in 1994, the multiplicative decomposition $\boldsymbol{F}=\boldsymbol{F}_{\mathrm{e}} \boldsymbol{F}_{\mathrm{g}}$ of the deformation gradient $\boldsymbol{F}$ into a growth part $\boldsymbol{F}_{\mathrm{g}}$ and an elastic part $\boldsymbol{F}_{\mathrm{e}}$ is nowadays a common approach for the description of growth in soft biological tissues. The growth deformation is assumed to be stress-free, such that stresses arise only from the elastic part $\boldsymbol{C}_{\mathrm{e}}=\boldsymbol{F}_{\mathrm{g}}^{-T} \boldsymbol{C F}_{\mathrm{g}}^{-1}$ of the strains. Utilizing constitutive equations for hyperelastic materials, the stress tensor $\boldsymbol{S}_{\mathrm{e}}$ in the intermediate configuration can be computed from $\boldsymbol{S}_{\mathrm{e}}=2 \frac{\partial \psi}{\partial \boldsymbol{C}_{\mathrm{e}}}$. Here, the formulation for fiber-reinforced soft biological tissues by Balzani et al. 9] is applied, whereby polyconvexity of the strain energy function and thus ellipticity and existence of minimizers are ensured.

The growth behavior is governed by the specific form of the growth tensor, which involves one or several local variables referred to as growth factors $\vartheta_{i}$. The evolution equations for these growth factors $\dot{\vartheta}_{i}=k_{\vartheta}\left(\vartheta_{i}\right) \phi_{i}\left(\boldsymbol{Z}_{i}\right)$, are formulated as function of the growth-driving quantity $\phi\left(\boldsymbol{Z}_{i}\right)$ and the growth function $k_{\vartheta}$ including the time-dependency. 
In this section, a model for anisotropic growth, relating the growth tensor $\boldsymbol{F}_{\mathrm{g}}$ to the anisotropy directions of the fibrous tissue material, is presented in Subsection 2.1. Applying this framework to idealized arterial segments under internal pressure, residual stress distributions that qualitatively reflect experimental observations can be generated. In Subsection 2.2. the focus is directed on the numerical calculation of the deformations which arise from resecting and slicing previously simulated, residually stressed arterial segments.

\subsection{MODEL OF ANISOTROPIC GROWTH RELATED TO TWO FIBER FAMILIES}

An effective approach to reduce stresses is the enlargement of the cross-sectional area. Accounting for this, the basic form $\boldsymbol{F}_{\mathrm{g}}=\vartheta \boldsymbol{I}+(1-\vartheta) \boldsymbol{A} \otimes \boldsymbol{A}$, describing a deformation with factor $\vartheta$ in all directions perpendicular to the direction given by the vector $\boldsymbol{A}$, is chosen among different existing approaches for the growth tensor, see e. g. [10].

In constitutive material models for arterial tissues, two main fiber directions $\boldsymbol{A}^{(a)}$ with $a=1,2$ are usually considered to approximate the complex layer-wise arrangement of collagen fibers. Assuming the anisotropy of growth to be linked with the fibrous tissue composition, a multiplicative decomposition of the growth tensor

$$
\boldsymbol{F}_{\mathrm{g}}=\boldsymbol{F}_{\mathrm{g}}^{(\mathrm{II})} \boldsymbol{F}_{\mathrm{g}}^{(\mathrm{I})}
$$

into two separated parts

$$
\begin{aligned}
\boldsymbol{F}_{\mathrm{g}}^{(a)} & =\vartheta^{(a)} \boldsymbol{I}+\left(1-\vartheta^{(a)}\right) \boldsymbol{M}^{(a)} \text { with } \\
\boldsymbol{M}^{(a)} & =\boldsymbol{A}_{\mathrm{g}}^{(a)} \otimes \boldsymbol{A}_{\mathrm{g}}^{(a)}, \quad a=\mathrm{I}, \mathrm{II}
\end{aligned}
$$

is considered in order to account for two different anisotropy directions defined by the vectors $\boldsymbol{A}_{\mathrm{g}}^{(\mathrm{I})}$ and $\boldsymbol{A}_{\mathrm{g}}^{(\mathrm{II})}$. This formulation is based on the assumption that in arterial walls, even in patient-specific ones, a stress state exists with one negative principle stress showing in radial direction and two positive principle stresses in a plane of tensile loading (which coincides with the axial-circumferential plane in perfect-tube arteries). This can be supposed to be the reason for the observation that mainly two collagen fiber families, oriented in directions within this plane, occur in arterial walls. The resulting tissue structure might enable an optimized load-bearing mechanism, cf. [11, 12. In more detail, these two fiber families may favorably be oriented symmetrically with respect to the direction of the highest principal stresses and thus, growth perpendicular to these directions would be desired by the tissue to reduce the stresses. Consequently, the growth orientation vectors $\boldsymbol{A}_{\mathrm{g}}^{(a)}$ are defined as angle bisectors of the fiber direction vectors according to

$$
\begin{aligned}
\boldsymbol{A}_{\mathrm{g}}^{(\mathrm{I})} & =\frac{\boldsymbol{A}^{(1)}+\boldsymbol{A}^{(2)}}{\left\|\boldsymbol{A}^{(1)}+\boldsymbol{A}^{(2)}\right\|} \text { and } \\
\boldsymbol{A}_{\mathrm{g}}^{(\mathrm{II})} & =\frac{\boldsymbol{A}^{(1)}-\boldsymbol{A}^{(2)}}{\left\|\boldsymbol{A}^{(1)}-\boldsymbol{A}^{(2)}\right\|} .
\end{aligned}
$$

For the determination of the growth factors $\vartheta^{(a)}$, two evolution equations

$$
\dot{\vartheta}^{(a)}=k_{\vartheta}\left(\vartheta^{(a)}\right) \phi^{(a)}\left(\boldsymbol{Z}^{(a)}\right), \quad a=\mathrm{I}, \mathrm{II}
$$

have to be considered. Therein, the function

$$
k_{\vartheta}\left(\vartheta^{(a)}\right)= \begin{cases}k_{\vartheta}^{+}\left[\frac{\vartheta^{+}-\vartheta^{(a)}}{\vartheta^{+}-1}\right]^{m_{\vartheta}^{+}}, \quad \phi^{(a)}>0 \\ k_{\vartheta}^{-}\left[\frac{\vartheta^{(a)}-\vartheta^{-}}{1-\vartheta^{-}}\right]^{m_{\vartheta}^{-}}, \quad \phi^{(a)}<0,\end{cases}
$$

which has been adopted from Lubarda \& Hoger [13], scales the growth velocity by a factor $k_{\vartheta}^{+/-}$and restricts the range of the growth factors to $\left[\vartheta^{-}, \vartheta^{+}\right]$. The function $\phi^{(a)}\left(\boldsymbol{Z}^{(a)}\right)$ defines the quantity which induces growth. Recent publications examining stressdriven growth [6, 7, 14 propagate the utilization of the trace of the elastic part of the Mandel stress given by $\phi\left(\boldsymbol{C}_{\mathrm{e}} \boldsymbol{S}_{\mathrm{e}}\right)=\boldsymbol{C}_{\mathrm{e}} \boldsymbol{S}_{\mathrm{e}}: \boldsymbol{I}$ as measure for the pressure acting on the arterial wall. Since this quantity is isotropic, it appears not to be adequate in combination with the anisotropic growth tensor and is therefore modified as follows. Using the projections of the elastic part of the Mandel stress into the anisotropy directions $\boldsymbol{A}_{\mathrm{g}}^{(a)}$,

$$
\begin{aligned}
\phi^{(a)}\left(\boldsymbol{C}_{\mathrm{e}} \boldsymbol{S}_{\mathrm{e}}\right) & =\boldsymbol{C}_{\mathrm{e}} \boldsymbol{S}_{\mathrm{e}}: \boldsymbol{M}_{\mathrm{e}}^{(a)} \\
\text { with } \quad \boldsymbol{M}_{\mathrm{e}}^{(a)} & =\boldsymbol{A}_{\mathrm{g}}^{(a)} \otimes \boldsymbol{A}_{\mathrm{g}}^{(a)},
\end{aligned}
$$

it can be ensured that growth in the plane perpendicular to $\boldsymbol{A}_{\mathrm{g}}^{(a)}$ is only emerging if the stress in this direction is nonzero. The index " $\mathrm{e}$ " indicates, that the structural tensors $\boldsymbol{M}_{\mathrm{e}}^{(a)}$ refer to the intermediate configuration. However, being eigenvectors of the growth tensor, the growth orientation vectors $\boldsymbol{A}_{\mathrm{g}}^{(a)}$ do not change their direction during the transformation to the intermediate configuration, thus $\boldsymbol{M}_{\mathrm{e}}^{(a)}=\boldsymbol{M}^{(a)}$.

Involving strain and stress quantites in the intermediate configuration, i. e. $\boldsymbol{C}_{\mathrm{e}}=\boldsymbol{F}_{\mathrm{g}}^{-T} \boldsymbol{C F}_{\mathrm{g}}^{-1}$ and $\boldsymbol{S}_{\mathrm{e}}=2 \frac{\partial \psi}{\partial \boldsymbol{C}_{\mathrm{e}}}$, the functions $\phi^{(a)}$ depend on both growth factors $\vartheta^{(a)}$, yielding a coupled system of evolution equations (6). Using an Euler backward scheme as for instance done in [7, the approximations

$$
\dot{\vartheta}^{(a)} \approx \frac{\vartheta_{n+1}^{(a)}-\vartheta_{n}^{(a)}}{\Delta t}, \quad a=\mathrm{I}, \mathrm{II}
$$

are adopted in order to compute the growth factors belonging to the actual time step $n+1$. Note that the index $n+1$ is skipped from now on and only 
quantities at the last time step are denoted by the index $n$. Consequently, the local residuals

$$
\begin{aligned}
R^{(\mathrm{I})} & :=-\vartheta^{(\mathrm{I})}+\vartheta_{n}^{(\mathrm{I})}+k_{\vartheta}\left(\vartheta^{(\mathrm{I})}\right) \phi^{(\mathrm{I})}\left(\vartheta^{(\mathrm{I})}, \vartheta^{(\mathrm{II})}\right) \Delta t, \\
R^{(\mathrm{II})} & :=-\vartheta^{(\mathrm{II})}+\vartheta_{n}^{(\mathrm{II})}+k_{\vartheta}\left(\vartheta^{(\mathrm{II})}\right) \phi^{(\mathrm{II})}\left(\vartheta^{(\mathrm{I})}, \vartheta^{(\mathrm{II})}\right) \Delta t
\end{aligned}
$$

have to fulfill $R^{(\mathrm{I})}=R^{(\mathrm{II})}=0$. To solve these nonlinear equations, a local Newton iteration, characterized by index $k$, is performed. The linearized residuals

$$
\begin{gathered}
\operatorname{Lin} R^{(\mathrm{I})}=\left.R^{(\mathrm{I})}\right|_{\vartheta(a), k}+\left.\frac{\partial R^{(\mathrm{I})}}{\partial \vartheta^{(\mathrm{I})}}\right|_{\vartheta^{(a), k}} \Delta \vartheta^{(\mathrm{I}), k+1}+\ldots \\
\left.\ldots \frac{\partial R^{(\mathrm{I})}}{\partial \vartheta^{(\mathrm{II})}}\right|_{\vartheta^{(a), k}} \Delta \vartheta^{(\mathrm{II}), k+1}=0, \\
\operatorname{Lin} R^{(\mathrm{II})}=\left.R^{(\mathrm{II})}\right|_{\vartheta^{(a), k}}+\left.\frac{\partial R^{(\mathrm{II})}}{\partial \vartheta^{(\mathrm{I})}}\right|_{\vartheta^{(a), k}} \Delta \vartheta^{(\mathrm{I}), k+1}+\ldots \\
\left.\ldots \frac{\partial R^{(\mathrm{II})}}{\partial \vartheta^{(\mathrm{II})}}\right|_{\vartheta^{(a), k}} \Delta \vartheta^{(\mathrm{II}), k+1}=0
\end{gathered}
$$

are solved for the incremental updates $\Delta \vartheta^{(\mathrm{I})}$ and $\Delta \vartheta^{(\mathrm{II})}$, and $\vartheta^{(\mathrm{I})}$ and $\vartheta^{(\mathrm{II})}$ are updated until an appropriate abort criterion is fulfilled. During this iteration, the incremental updates are calculated by

$$
\begin{aligned}
\Delta \vartheta^{(\mathrm{I}), k+1} & =\left.\frac{R^{(\mathrm{I})}}{A_{\vartheta}^{(\mathrm{I}, \mathrm{I})}}\right|_{\vartheta^{(a), k}}-\left.\frac{A_{\vartheta}^{(\mathrm{I}, \mathrm{II})}}{A_{\vartheta}^{(\mathrm{I}, \mathrm{I})}}\right|_{\vartheta^{(a), k}} \Delta \vartheta^{(\mathrm{II}), k+1}, \\
\Delta \vartheta^{(\mathrm{II}), k+1} & =\left.\frac{A_{\vartheta}^{(\mathrm{I}, \mathrm{I})} R^{(\mathrm{II})}-A_{\vartheta}^{(\mathrm{II}, \mathrm{I})} R^{(\mathrm{I})}}{A_{\vartheta}^{(\mathrm{I}, \mathrm{I})} A_{\vartheta}^{(\mathrm{II}, \mathrm{II})}-A_{\vartheta}^{(\mathrm{I}, \mathrm{II})} A_{\vartheta}^{(\mathrm{II}, \mathrm{I})}}\right|_{\vartheta^{(a), k}},
\end{aligned}
$$

where the abbreviations

$$
\begin{aligned}
A_{\vartheta}^{(\mathrm{I}, \mathrm{I})} & =-\frac{\partial R^{(\mathrm{I})}}{\partial \vartheta^{(\mathrm{I})}}, & A_{\vartheta}^{(\mathrm{I}, \mathrm{II})} & =-\frac{\partial R^{(\mathrm{I})}}{\partial \vartheta^{(\mathrm{II})}}, \\
A_{\vartheta}^{(\mathrm{II}, \mathrm{I})} & =-\frac{\partial R^{(\mathrm{II})}}{\partial \vartheta^{(\mathrm{I})}}, & A_{\vartheta}^{(\mathrm{II}, \mathrm{II})} & =-\frac{\partial R^{(\mathrm{II})}}{\partial \vartheta^{(\mathrm{II})}}
\end{aligned}
$$

are used. Thus, the derivatives of the functions $k_{\vartheta}$ and $\phi^{(a)}$ with respect to the growth factors have to be provided. Differentiating eqs. (7) and (8) leads to

$$
\frac{\partial k_{\vartheta}\left(\vartheta^{(a)}\right)}{\partial \vartheta^{(a)}}=\left\{\begin{array}{l}
\frac{m_{\vartheta}^{+}}{\vartheta^{(a)}-\vartheta^{+}} k_{\vartheta}\left(\vartheta^{(a)}\right), \phi^{(a)}>0 \\
\frac{m_{\vartheta}^{-}}{\vartheta^{(a)}-\vartheta^{-}} k_{\vartheta}\left(\vartheta^{(a)}\right), \phi^{(a)}<0
\end{array}\right.
$$

and

$$
\begin{aligned}
\frac{\partial \phi^{(i)}}{\partial \vartheta^{(j)}} & =\frac{\partial \boldsymbol{C}_{\mathrm{e}} \boldsymbol{S}_{\mathrm{e}}}{\partial \vartheta^{(j)}}: \boldsymbol{M}_{\mathrm{e}}^{(i)} \\
& =\left(\frac{\partial \boldsymbol{C}_{\mathrm{e}}}{\partial \vartheta^{(j)}} \boldsymbol{S}_{\mathrm{e}}+\boldsymbol{C}_{\mathrm{e}} \frac{\partial \boldsymbol{S}_{\mathrm{e}}}{\partial \vartheta^{(j)}}\right): \boldsymbol{M}_{\mathrm{e}}^{(i)} \\
& =\left(\frac{\partial \boldsymbol{C}_{\mathrm{e}}}{\partial \vartheta^{(j)}} \boldsymbol{S}_{\mathrm{e}}+\boldsymbol{C}_{\mathrm{e}}\left(\frac{1}{2} \hat{\mathbb{C}}^{\mathrm{e}}: \frac{\partial \boldsymbol{C}_{\mathrm{e}}}{\partial \vartheta^{(j)}}\right)\right): \boldsymbol{M}_{\mathrm{e}}^{(i)}
\end{aligned}
$$

with $i=\mathrm{I}$, II and $j=\mathrm{I}$, II. Herein, $\hat{\mathbb{C}}^{\mathrm{e}}=2 \frac{\partial \boldsymbol{S}_{\mathrm{e}}}{\partial \boldsymbol{C}_{\mathrm{e}}}$ is the elastic part of the tangent moduli in the intermediate configuration. The remaining unknown derivative of the strain $\boldsymbol{C}_{\mathrm{e}}$ with respect to the growth factor $\vartheta^{(a)}$ has to be computed according to the chain rule by

$$
\frac{\partial \boldsymbol{C}_{\mathrm{e}}}{\partial \vartheta^{(a)}}=\frac{\partial \boldsymbol{C}_{\mathrm{e}}}{\partial \boldsymbol{F}_{\mathrm{g}}}: \frac{\partial \boldsymbol{F}_{\mathrm{g}}}{\partial \vartheta^{(a)}}=\frac{\partial \boldsymbol{C}_{\mathrm{e}}}{\partial \boldsymbol{F}_{\mathrm{g}}}: \frac{\partial \boldsymbol{F}_{\mathrm{g}}}{\partial \boldsymbol{F}_{\mathrm{g}}^{(a)}}: \frac{\partial \boldsymbol{F}_{\mathrm{g}}^{(a)}}{\partial \vartheta^{(a)}}
$$

The first part is given through

$$
\frac{\partial \boldsymbol{C}_{\mathrm{e}}}{\partial \boldsymbol{F}_{\mathrm{g}}}=-\boldsymbol{F}_{\mathrm{g}}^{-T} \otimes \boldsymbol{C}_{\mathrm{e}}-\boldsymbol{C}_{\mathrm{e}} \otimes \boldsymbol{F}_{\mathrm{g}}^{-T}
$$

and the relations

$$
\begin{aligned}
\frac{\partial \boldsymbol{F}_{\mathrm{g}}}{\partial \boldsymbol{F}_{\mathrm{g}}^{(\mathrm{I})}} & =\boldsymbol{F}_{\mathrm{g}}^{(\mathrm{II})} \otimes \boldsymbol{I}, \\
\frac{\partial \boldsymbol{F}_{\mathrm{g}}}{\partial \boldsymbol{F}_{\mathrm{g}}^{(\mathrm{II})}} & =\boldsymbol{I} \otimes\left(\boldsymbol{F}_{\mathrm{g}}^{(\mathrm{I})}\right)^{T}, \\
\frac{\partial \boldsymbol{F}_{\mathrm{g}}^{(a)}}{\partial \vartheta^{(a)}} & =\boldsymbol{I}-\boldsymbol{M}^{(a)}, \quad a=\mathrm{I}, \mathrm{II}
\end{aligned}
$$

can be derived for the other parts ${ }^{1}$ Combining eqs. 17)/ 18 and (19), the results

$$
\begin{gathered}
\frac{\partial \boldsymbol{F}_{\mathrm{g}}}{\partial \vartheta^{(\mathrm{I})}}=\boldsymbol{F}_{\mathrm{g}}^{(\mathrm{II})}-\boldsymbol{F}_{\mathrm{g}}^{(\mathrm{II})} \boldsymbol{M}^{(\mathrm{I})}, \\
\frac{\partial \boldsymbol{F}_{\mathrm{g}}}{\partial \vartheta^{(\mathrm{II})}}=\boldsymbol{F}_{\mathrm{g}}^{(\mathrm{I})}-\boldsymbol{M}^{(\mathrm{II})} \boldsymbol{F}_{\mathrm{g}}^{(\mathrm{I})}
\end{gathered}
$$

are obtained and all required parts for the incremental updates of the growth factors in eqs. (11) - 12) are determined.

Given the final values of $\vartheta^{\mathrm{I}}$ and $\vartheta^{\mathrm{II}}$, the $2^{\text {nd }}$ PiolaKirchhoff stress $\boldsymbol{S}=\boldsymbol{F}_{\mathrm{g}}^{-1} \boldsymbol{S}_{\mathrm{e}} \boldsymbol{F}_{\mathrm{g}}^{-T}$ can be identified performing a pull back of $\boldsymbol{S}_{\mathrm{e}}$ to the reference configuration.

\subsection{VisuAlization OF RESIDUAL STRESSES}

Anticipating the results of the numerical example in Section 3, where an idealized arterial segment under constant internal pressure is examined using Finite Element calculations, the question arises, how to determine the deformations which are related to residual stress states. In order to permit these deformations, a change of the boundary conditions is required, for instance the release of the right edge of a residually stressed arterial segment as shown in Figure 1, which enables the opening of the segment by the angle $\varphi$.

Dealing with highly nonlinear material behavior and large deformations, this final deformation state can not be determined in one calculation step, thus a special procedure has to be applied. Here, this procedure is proposed as follows.

$$
\begin{aligned}
& { }^{1} \text { The symbol } \otimes \text { is defined such that the relation } \\
& \underbrace{(\boldsymbol{A} \otimes \boldsymbol{B})}_{=: \mathbb{D}}:(\boldsymbol{a} \otimes \boldsymbol{b})=\boldsymbol{A} \boldsymbol{a} \otimes \boldsymbol{B} \boldsymbol{b}, \quad \mathbb{D}_{i j k l}=A_{i k} B_{j l}
\end{aligned}
$$

is valid, which for instance results in the calculation rule $A B C=\left(A \otimes C^{T}\right): B$. 


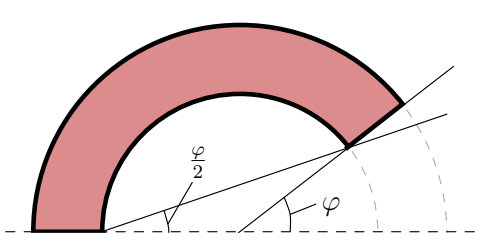

Figure 1. Definition of the opening angle $\varphi$ in an idealized, symmetric arterial geometry.

(1.) Define the initial boundary value problem including the discretization of the unstressed configuration, apply the loads stepwise and hold them constant until a growth equilibrium state is reached.

(2.) Deactivate growth and remove the loads stepwise to determine the residually stressed state. Due to growth, the obtained geometry differs from the initial reference configuration.

(3.) Save this grown, unloaded configuration by storing the nodal coordinates as well as the fiber orientation vectors and the residual Cauchy stress $\boldsymbol{\sigma}_{\text {res }}:=(\operatorname{det} \boldsymbol{F})^{-1} \boldsymbol{F} \boldsymbol{S} \boldsymbol{F}^{T}$ at the integration points.

(4.) Use the saved nodal coordinates and fiber orientation vectors to define a new boundary value problem with boundary conditions that allow for the expected deformation.

(5.) Restore $\boldsymbol{\sigma}_{\text {res }}$ at each integration point and keep it throughout the following calculation.

(6.) Apply the residual stresses internally by updating the stress tensor according to $\boldsymbol{S} \leftarrow \boldsymbol{S}+f_{\text {load }} \boldsymbol{S}_{\text {res }}$, where $f_{\text {load }}$ is a load factor ranging from 0 to 1 , while growth is deactivated. In each step, the stress $\boldsymbol{S}_{\text {res }}=\operatorname{det}[\boldsymbol{F}] \boldsymbol{F}^{-1} \boldsymbol{\sigma}_{\text {res }} \boldsymbol{F}^{-T}$ has to be computed corresponding to the actual deformation state.

Following this procedure, the wanted deformation state will arise in absence of external loads.

\section{NumericAl EXAMPLE}

In a previous comparative study [15], an arterial segment with internal pressure and an axial displacement of $15 \%$ of the initial length was considered for a comparison of two different forms of the growth tensor and two different driving forces. Each of the approaches was able to provoke growth-induced circumferential stress smoothening, but the approach presented in Subsection 2.1 turned out to be the most effective, generating the most beneficial stress distributions at the lowest growth requirement, measured by the determinant of the growth tensor. Therefore, a similar boundary value problem making use of this approach will be analyzed here in more detail, focussing in particular on the simulation of the opening artery.

The half of a one-layered idealized arterial segment, reinforced by two fiber families arranged with angles of $\pm 30^{\circ}$ with respect to the circumferential direction, is exposed to an internal pressure of $16 \mathrm{kPa}(120 \mathrm{mmHg})$. As an approximation of the natural conditions in the

\begin{tabular}{ccccc}
\hline$c_{1}[\mathrm{kPa}]$ & $\epsilon_{1}[\mathrm{kPa}]$ & $\epsilon_{2}[-]$ & $\alpha_{1}[\mathrm{kPa}]$ & $\alpha_{2}[-]$ \\
\hline 17.5 & 499.8 & 2.4 & 30001.9 & 5.1 \\
\hline
\end{tabular}

TABLE 1. Material parameters for arterial tissue, adjusted by Brands et al. 16 to the model from 9].

\begin{tabular}{cccccc}
\hline$\vartheta^{+}[-]$ & $k_{\vartheta}^{+}[-]$ & $m_{\vartheta}^{+}[-]$ & $\vartheta^{-}[-]$ & $k_{\vartheta}^{-}[-]$ & $m_{\vartheta}^{-}[-]$ \\
\hline 1.1 & 1.0 & 3.0 & 0.9 & 1.0 & 3.0 \\
\hline
\end{tabular}

TABLE 2. Growth parameters used for the simulation of an idealized arterial segment.

body, an axial displacement is additionally applied and three different values, namely $10 \%, 15 \%$ and $20 \%$ of the initial length, are compared. The material and growth parameters are chosen as given in Tables 1 and 2 , respectively. The load is applied within $1 \mathrm{~s}$ and subsequently held constant until $t=12 \mathrm{~s}$, letting the arterial segment grow.

In Figure 2 the resulting stress distributions (solid lines) are shown and compared with the stress distributions obtained without including growth (dashed lines). For each value of axial strain, the circumferential stress distribution is significantly smoothened, which reduces the stress peak at the inner surface. The axial stress distributions are smoothened as well and moreover, a distinct stress reduction can be observed which is a consequence of the axial component of growth, compensating the axial deformation. The final axial stress level differs strongly in dependence on the axial displacement: the higher the axial displacement, the higher the axial stresses. Examining the growth deformation in terms of the determinant of the growth tensor, depicted in the contour plots in Figure 2, it can be observed that the volume change due to growth increases slightly with increasing axial displacement, being nevertheless in the same range.

Removing the internal pressure while maintaining the axial displacement, the stress distributions given in Figure 3 are obtained. Being thus not free of external loads in the axial direction, there are non-zero axial stresses in the non-growing reference segments in this state. In the growing segments, non-zero circumferential and axial stresses occur, which are - despite the existence of the axial load - referred to as residual stresses in this contribution. Showing circumferential tensile stresses at the outer surface, each of the considered arterial segments is expected to open after a radial cut. The axial stresses in the segments are positive as well, provoking an axial contraction when releasing the axial constraint.

The deformations related to these operations are depicted in Figure 4 Depending on the axial displacement, different opening angles and maximal values of axial strain are obtained. With increasing axial displacement, the opening angle reduces and the axial contraction rises. In the opened segments, the circumferential stresses have vanished. 


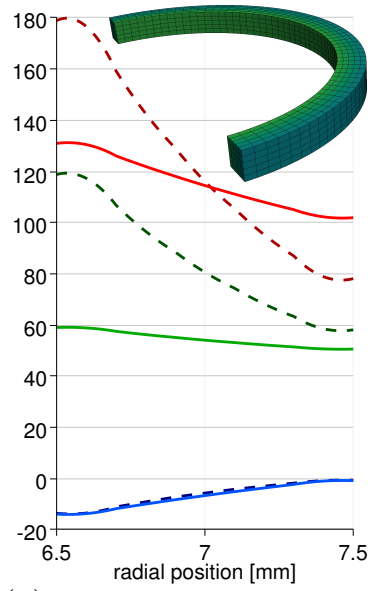

(a)

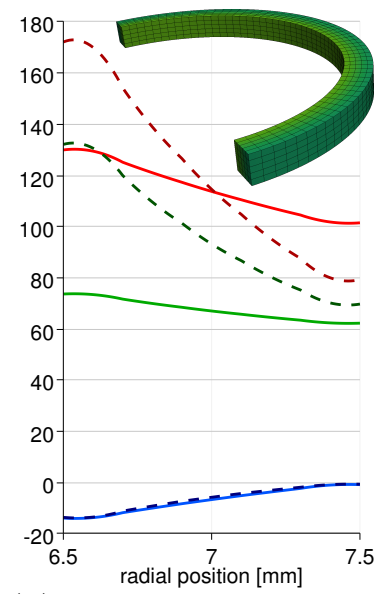

(b)

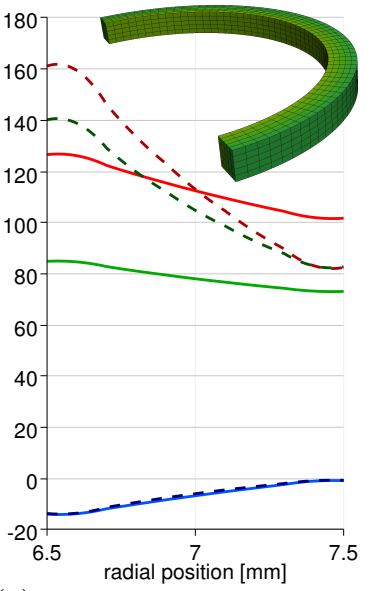

(c)

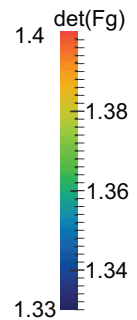

- radial stress $[\mathrm{kPa}]$

- circumf. stress [kPa]

- axial stress $[\mathrm{kPa}]$

- - radial stress, no growth

- - circumf. stress, no growth

- - axial stress, no growth

FiguRE 2. Radial, circumferential and axial stress distributions as well as growth deformation at $t=12 \mathrm{~s}$ in an arterial segment exposed to an internal pressure of $16 \mathrm{kPa}$ and different axial displacements of (a) $10 \%$, (b) $15 \%$ and (c) $20 \%$ of the initial length. The dashed lines indicate the stresses of a non-growing arterial segment.

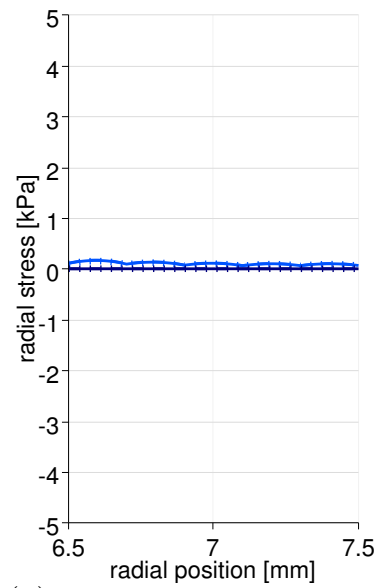

(a)

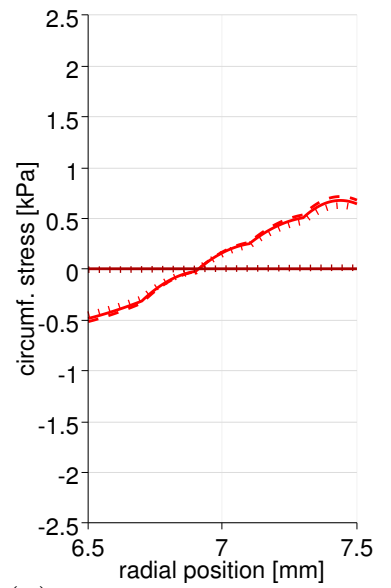

(b)

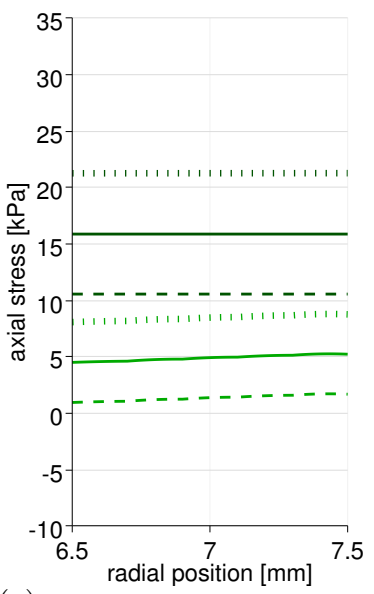

(c)

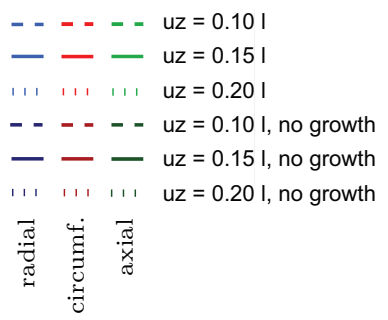

Figure 3. Residual stresses in (a) radial and (b) circumferential direction, as well as (c) stresses in axial direction at $t=12 \mathrm{~s}$ after removing the internal pressure while keeping up the axial displacement. The stress distributions provoke the axial contraction and opening of a resected, radially sliced arterial segment as it is observed in experiments.

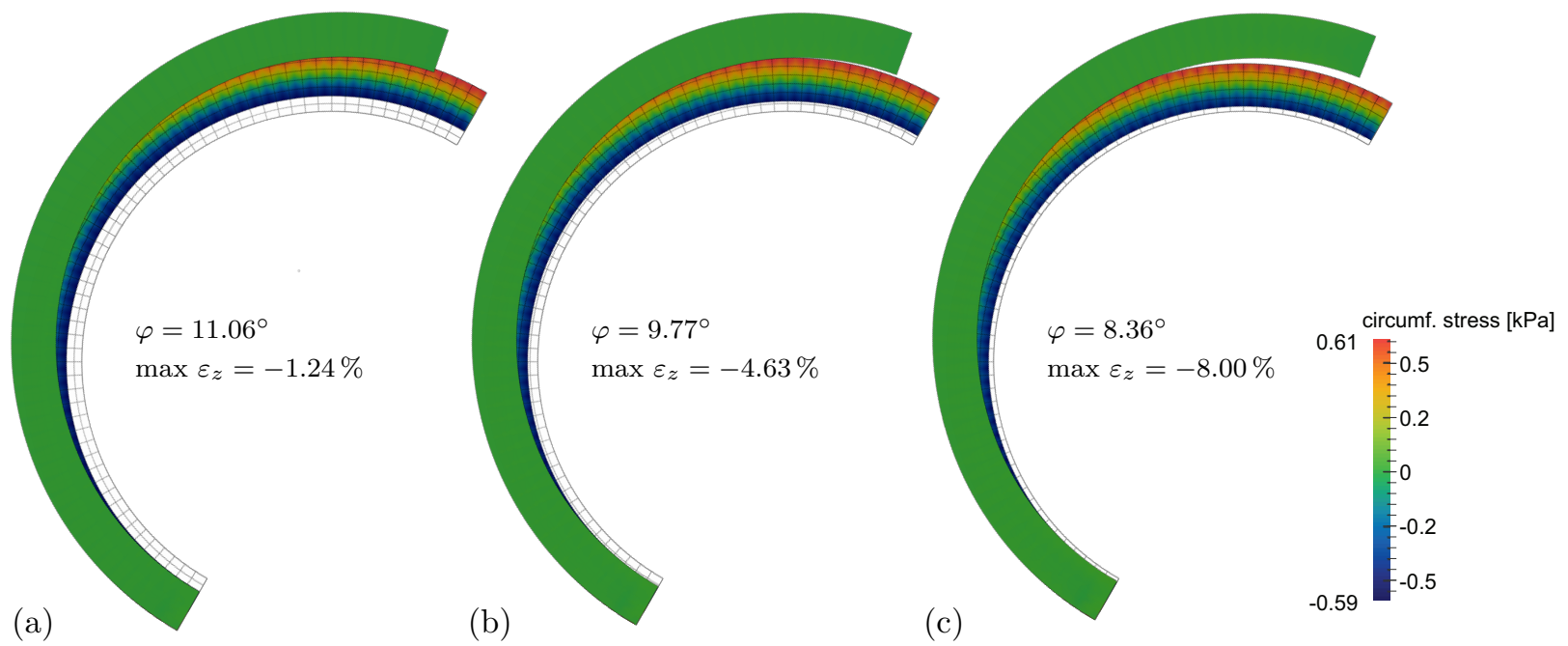

Figure 4. Deformation states associated to the residual stresses in arterial segments loaded by an internal pressure and axial displacements of (a) $10 \%$, (b) $15 \%$ and (c) $20 \%$ of the initial length. The wireframe shows the undeformed reference configuration, whereas the second chart form the inside shows the grown state after removal of the pressure. The indicated values of axial strain have been computed with respect to that deformed configuration. 


\section{Discussion and Conclusion}

Generating favorable results in specific cases as seen in Section 3, the performance of the presented model is still strongly dependent on the boundary value problem, especially on the choice of the fiber orientation vectors and on the boundary conditions. The approximation of realistic conditions is difficult for both issues.

Subjected to remodeling processes, fibers in arterial tissue reorient according to the requirements of their mechanobiological environment. This effect of improving the load-bearing behavior is not included in the current framework so far, such that remodeling caused changes of the growth-inducing stresses and their consequences on growth can not be considered. Therefore, the interaction between growth and remodeling processes is a topic which should be taken into account to further improve the model.

The axial boundary conditions assumed in this contribution are a vague approach to approximate the natural state of arteries in the body, where axial strains and stresses are existent. Thus, one could also have decided to apply axial stresses instead, unknowing which is the best way to mimic the unmeasurable, real conditions.

Another point with respect to the axial direction shall be mentioned. In contrast to the circumferential direction, where unloading and slicing are two different operations, the compution af axial residual stresses is tricky and demands further meditation. To be able to simulate the contraction after resecting the artery, the applied axial displacement has not been removed in the presented numerical example when computing the "residually" stressed state. Doing so, the requested release of the axial constraint could be performed in the modified boundary value problem. If indeed real residual stresses are the matter of interest, the axial loading has to be removed together with the internal pressure, which is only possible when applying an axial stress instead of a displacement. Then, the arterial segment can already deform in axial direction during the unloading process, such that no further cutting, revealing the deformation associated to axial residual stresses, is possible afterwards.

A third topic for further examinations is the development of a procedure to adjust the growth parameters, which have been chosen arbitrarily until now.

\section{ACKNOWLEDGEMENTS}

The authors appreciate funding from DFG through the institutional strategy "The Synergetic University" of TU Dresden.

\section{REFERENCES}

[1] T. Matsumoto, K. Hayashi. Response of arterial wall to hypertension and residual stress. In K. Hayashi, A. Kamiya, K. Ono (eds.), Biomechanics. Springer, 1996.

[2] D. Balzani, J. Schröder, D. Gross. Simulation of discontinuous damage incorporating residual stresses in circumferentially overstretched atherosclerotic arteries. Acta Biomaterialia 2:609-618, 2006.

DOI:10.1016/j.actbio.2006.06.005

[3] H. Weisbecker, D. M. Pierce, G. A. Holzapfel. A generalized prestressing algorithm for finite element simulations of preloaded geometries with application to the aorta. Int $J$ Numer Method Biomed Eng 30:857-872, 2014. DOI:10.1002/cnm.2632

[4] J. Schröder, S. Brinkhues. A novel scheme for the approximation of residual stresses in arterial walls. Archive of Applied Mechanics 84:881-898, 2014. DOI:10.1007/s00419-014-0838-x.

[5] J. Schröder, M. von Hoegen. An engineering tool to estimate eigenstresses in three-dimensional patientspecific arteries. Comput Methods Appl Mech Engrg 306:364-381, 2016. DOI:10.1016/j.cma.2016.03.020

[6] S. Göktepe, O. J. Abilez, E. Kuhl. A generic approach towards finite growth with examples of athlete's heart, cardiac dilation, and cardiac wall thickening. Journal of the Mechanics and Physics of Solids 58:1661-1680, 2010. DOI:10.1016/j.jmps.2010.07.003

[7] P. Sáez, E. Peña, M. A. Martínez, E. Kuhl. Computational modeling of hypertensive growth in the human carotid artery. Computational Mechanics 53:1183-1196, 2014. DOI:10.1007/s00466-013-0959-z

[8] E. K. Rodriguez, A. Hoger, A. D. McCulloch. Stress-dependent finite growth in soft elastic tissues. Journal of Biomechanics 27(4):455-467, 1994. DOI:10.1016/0021-9290(94)90021-3

[9] D. Balzani, P. Neff, J. Schröder, G. A. Holzapfel. A polyconvex framework for soft biological tissues. adjustment to experimental data. Int J Solids Struct 43:6052-6070, 2006. DOI:10.1016/j.ijsolstr.2005.07.048

[10] E. Kuhl. Growing matter: A review of growth in living systems. J Mech Behav Biomed Mater 29:529-543, 2014. DOI:10.1016/j.jmbbm.2013.10.009

[11] I. Hariton, G. deBotton, T. C. Gasser, G. A. Holzapfel. Stress-driven collagen fiber remodeling in arterial walls. Biomechanics and Modeling in Mechanobiology 6:163-175, 2007. DOI:10.1007/s10237-006-0049-7.

[12] S. Fausten, D. Balzani, J. Schröder. An algorithmic scheme for the automated calculation of fiber orientations in arterial walls. Computational Mechanics 2016. DOI:10.1007/s00466-016-1321-z.

[13] V. A. Lubarda, A. Hoger. On the mechanics of solids with a growing mass. Int $J$ Solids Struct 39:4627-4664, 2002. DOI:10.1016/S0020-7683(02)00352-9.

[14] E. Kuhl, R. Maas, G. Himpel, A. Menzel. Computational modeling of arterial wall growth. Biomechanics and Modeling in Mechanobiology 6:321-331, 2007. DOI:10.1007/s10237-006-0062-x.

[15] A. Zahn, D. Balzani. Modeling residual stresses in arterial walls based on anisotropic growth. Proceedings in Applied Mathematics and Mechanics 16:115-116, 2016. DOI:10.1002/pamm.201610046

[16] D. Brands, A. Klawonn, O. Rheinbach, J. Schröder. Modelling and convergence in arterial wall simulations using a parallel feti solution strategy. Computer Methods in Biomechanics and Biomedical Engineering 11:569-583, 2008. DOI:10.1080/10255840801949801 